\title{
RIESGO DE MALIGNIDAD EN CITOLOGÍA OBTENIDA MEDIANTE ASPIRACIÓN CON AGUJA FINA DE LA GLÁNDULA TIROIDES
}

\author{
MALIGNANCY RISK IN THE FINE NEEDLE ASPIRATION \\ CYTOLOGY OF THE THYROID GLAND
}

\author{
Osorio-Covo Carlos G. ${ }^{1}$ \\ Grice-Reyes Juan M. ${ }^{1}$ \\ Herrera-Banquez Karen ${ }^{1}$ \\ Caraballo-Gómez Cáceres Pedro ${ }^{1}$ \\ Agresott-Marsiglia Rubén ${ }^{2}$ \\ Redondo-De Oro Katherine ${ }^{3}$ \\ Herrera-Sáenz Francisco ${ }^{4}$
}

Correspondencia: fherreras@gruposcalpellum.com

Recibido para evaluación: agosto-25-2014. Aceptado para publicación: abril-23-2015.

\section{RESUMEN}

Introducción: un nódulo tiroideo puede ser la manifestación clínica tanto de una lesión benigna como maligna. La citología obtenida mediante aspiración con aguja fina es actualmente la prueba diagnóstica más costo efectiva para discriminar si se requieren manejo médico o quirúrgico.

Objetivo: identificar el riesgo de malignidad en la citología obtenida mediante aspiración con aguja fina en nódulos de la glándula tiroides, clasificado de acuerdo con el Sistema Bethesda para el reporte citopatológico.

Metodología: se llevó a cabo estudio retrospectivo con la revisión de historias clínicas de 790 pacientes que acudieron en el periodo comprendido entre enero del 2007 y diciembre de 2013 al E.S.E Hospital Universitario del Caribe, Cartagena, Colombia, con una patología tiroidea. Se seleccionaron 161 historias clínicas de pacientes sometidos a aspiración con aguja fina y luego sometidos a cirugía de tiroides. Debían tener disponibles los resultados de citología del aspirado y el estudio de anatomía patológica de la pieza operatoria. Se clasificó la citología de acuerdo con la categoría de diagnóstico citológico según el Sistema Bethesda para el Reporte Citopatologico de las Lesiones de la Glándula Tiroides. Se estimó el riesgo de malignidad para cada nivel de clasificación de la citología.

Resultados: el promedio de edad fue $43.9 \pm 13.7$ años. Los resultados obtenidos en la citología obtenida mediante aspiración con aguja fina fueron los siguientes: 12 (7.4\%) no diagnósticos, $109(67.70 \%)$ benignos, $2(1.2 \%)$ atipia folicular de significado indeterminado, $21(13.0 \%)$ sospechosos de neoplasia folicular, $9(5.5 \%)$ sospechosos de malignidad, y $8(4.9 \%)$ malignos. El riesgo de malignidad para cada una de las categorías de diagnóstico citológico fue el siguiente: $0.0 \%$ no diagnóstico, $11.0 \%$ y $5.5 \%$ benigno, incluyendo y excluyendo los microcarcinomas papilares respectivamente, $100 \%$ atipia folicular de significado indeterminado, $14.2 \%$ sospechoso de neoplasia folicular, $77.7 \%$ sospechoso de malignidad y $75 \%$ maligno.

\footnotetext{
${ }^{1}$ Estudiante de Medicina. Grupo de Investigación Scalpellum. Facultad de Medicina. Universidad de Cartagena. Colombia.

2 Médico. Estudiante de Postgrado. Cirugía General. Facultad de Medicina. Universidad de Cartagena. Grupo de Investigación Scalpellum. Cartagena. Colombia.

3 Médico. Patóloga. Docente Facultad de Medicina. Universidad de Cartagena. Grupo de Investigación Scalpellum. Cartagena. Colo,bia.

4 Médico. Especialista en Cirugía General. Docente Departamento Quirúrgico. Facultad de Medicina. Universidad de Cartagena. Director Grupo de Investigación Scalpellum. Colombia.
} 
ISSN: 2215-7840, 6(1), enero-junio 2015, Osorio-Covo Carlos G., Grice-Reyes Juan M., Herrera-Banquez Karen, Caraballo-Gómez Cáceres Pedro, Agresott-Marsiglia Rubén, Redondo-De Oro Katherine, Herrera-Sáenz Francisco

Conclusión: el riesgo de malignidad atribuible de los aspirados categorizados, en forma retrospectiva, de acuerdo al Sistema Bethesda para el Reporte Citopatologico de las lesiones de la Glándula Tiroides, fueron similares a los previamente descritos. Rev. ciec.biomed. 2015;6(1):22-28

PALABRAS CLAVE

Nódulo tiroideo; Citología; Biopsia con Aguja; Neoplasias de la Tiroides.

\section{SUMMARY}

Introduction: a thyroid nodule could be the clinical presentation so much of a benign as malign lesion. The fine needle aspiration cytology is currently the most effective diagnostic test to define if medical or surgical approach is required.

Objective: to identify the malignancy risk in the fine needle aspiration cytology in thyroid nodules, classified according to the Bethesda system for the cytopathological report.

Methods: a retrospective study was carried out with the review of clinical records of 790 patients who attended between January, 2007 and December, 2013 to the E.S.E Hospital Universitario del Caribe, Cartagena, Colombia, with a thyroid pathology. 161 clinical records of patients who received fine needle aspiration cytology and subsequently thyroid surgery were selected. They had to have available the cytopathological reports and the result of the tissue studied after the surgery. The cytology was classified according to the category of cytological diagnosis according to the Bethesda system for the cytopathological report of the lesions of the thyroid gland. A malignancy risk was estimated for each level of classification of the cytology.

Results: the average age was $43.9 \pm 13.7$ years. The obtained results in the fine needle aspiration cytology were: $12(7.4 \%)$ without diagnosis, $109(67.70 \%)$ were benign, $2(1.2 \%)$ indicated follicular atypia of indeterminate significance, $21(13.0 \%)$ were suspicious of follicular neoplasm, $9(5.5 \%)$ were suspected of malignancy and 8 $(4.9 \%)$ were malignant. The malignancy risk for each one of the categories of cytological diagnosis was: $0.0 \%$ non-diagnostic, $11.0 \%$ and $5.5 \%$ benign, including and excluding the papillary microcarcinoma respectfully, $100 \%$ follicular atypia of indeterminate significance, $14.2 \%$ with suspicious of follicular neoplasm, $77.7 \%$ with suspicious of malignancy and $75 \%$ malignant.

Conclusion: the malignancy risk attributable to the categorized aspirations, in a retrospective way, according to the Bethesda system for the cytopathological report of the lesions of thyroid gland were similar to those previously described. Rev.cienc. biomed. 2015;6(1):22-28

\section{KEYWORDS}

Thyroid nodule; Cytology; Biopsy needle; Thyroid neoplasms.

\section{INTRODUCCIÓN}

Los nódulos tiroideos constituyen un hallazgo frecuente en la práctica clínica. Su prevalencia en el examen físico mediante palpación puede variar entre $3-7 \%$ (1). Cuando la exploración se realiza mediante evaluación ecográfica, la prevalencia puede aumentar hasta el $76 \%$ (2), prevalencia que es similar a lo encontrado en autopsias, donde se reportan hallazgos entre 43$65 \%$, sin la existencia de patología tiroidea diagnosticada previamente en la historia clínica (1).

Un nódulo tiroideo puede ser manifestación de una lesión benigna o maligna. Es importante brindar un manejo adecuado, teniendo como esencia establecer si existe presencia potencial de malignidad (3). La citología obtenida mediante aspiración con aguja fina (CACAF) es actualmente el mejor método para la evaluación preoperatoria de los nódulos tiroideos (4). Antes del uso rutinario de la $C A C A F$, el porcentaje de nódulos tiroideos malignos que eran resecados correspondía aproximadamente al $14 \%$. Actualmente, con el uso generalizado de esta técnica, el porcentaje ha superado el 50\% (5).

Anteriormente las citológias obtenidas mediante aspiración con aguja fina eran reportadas de acuerdo a cuatro categorías diagnosticas: [A] insatisfactoria. [B] benigna. [C] sospechosa de malignidad. [D] francamente maligna. Sin embargo, la terminología varia- 
ba de un laboratorio a otro y se dificultaba la implementación homogénea de las conductas que los grupos quirúrgicos debía seguir (6). En el año 2009 fue publicado el Sistema Bethesda para el Reporte Citopatológico de las Lesiones de la Glándula Tiroides, cuyo objetivo principal consistió en estandarizar las definiciones citológicas y mejorar la comunicación entre patólogos y cirujanos. Este sistema recomienda reportar los diagnósticos citológicos en seis diferentes categorías: $[A]$ insatisfactorio. [B] benigno. [C] atipia folicular de significado indeterminado. [D] sospecha de neoplasia folicular. [E] sospecha de malignidad. [E] malignidad (7).

El estudio tiene como objetivo identificar el riesgo de malignidad en la citología por aspirado con aguja fina de nódulos tiroideos y clasificación de acuerdo con el Sistema Bethesda para el Reporte Citopatologico de las Lesiones de la Glándula Tiroides.

\section{MATERIALES Y MÉTODOS}

Determinación de la población de estudio. Se llevó a un estudio descriptivo de linealidad retrospectiva. Se identificaron las historias clínicas de 790 pacientes que acudieron a los servicios de endocrinología, cirugía general y otorrinolaringología durante el periodo comprendido entre enero del 2007 y diciembre del 2013, al Hospital Universitario del Caribe, Cartagena, Colombia, consultando por lesiones tiroideas. Los pacientes habían sido diagnosticados, de acuerdo a la décima versión de la Clasificación Internacional de Enfermedades (ClE-10). Las definiciones que se tuvieron en cuenta fueron: bocio coloide simple (E010), bocio coloide multinodular (E011), nódulo tiroideo solitario (E041), hipotiroidismo consecutivo a procedimientos (E089), neoplasia benigna de la glándula tiroides (D034) y neoplasia maligna de la glándula tiroides (C073).

Posteriormente se llevó a cabo la revisión de cada una de las historias. Se seleccionaron 161 pacientes en quienes fue realizada una CACAF y contaban con la descripción del estudio citológico de la aspiración, y posteriormente fueron sometidos a cirugía, contando con el estudio anatomopatológico del espécimen quirúrgico.
Distribución y criterios de diagnóstico citológico. Los diagnósticos citológicos fueron categorizados acuerdo con el Sistema Bethesda para el Reporte Citopatológico de las Lesiones Nodulares de la Glandula Tiroides. En los casos en que dicho diagnóstico no fue reportado utilizando el Sistema Bethes$\mathrm{da}$, se aplicaron las siguientes definiciones.

Bethesda I - No diagnóstico. Estudios citológicos en los que se reportaron los siguientes hallazgos: únicamente contenido quístico, espécimen prácticamente acelular, ausencia de material coloide, muestra con predominio de sangre y mala calidad de la fijación celular.

Bethesda II - Benigno. Estudios citológicos en los que se reportaron hallazgos consistentes con hiperplasia nodular, tiroiditis linfocítica y extendido con predominio de material coloide.

Bethesda III - Lesión folicular de significado indeterminado. Estudios citológicos que se reportaron como satisfactorios pero su diagnóstico no estaba definido $\mathrm{y} / \mathrm{o}$ el patólogo sugería nuevo aspirado de la lesión para revalorar.

Bethesda IV - Sospecha de neoplasia folicular. Estudios citológicos que reportaron hallazgos consistentes con moderada o abundante celularidad, ausencia o escasa cantidad de material coloide, células foliculares organizadas con configuración microfolicular o trabecular. Los casos en los que existía la sospecha de la presencia de células de Hürthle fueron incluidos en esta categoría.

Bethesda V - Sospecha de malignidad. Estudios citológicos que reportaron hallazgos sospechosos para carcinoma papilar de tiroides, carcinoma medular de tiroides, carcinoma anaplásico de tiroides, carcinoma metastásico extratiroideo o sospechoso para linfoma de origen tiroideo. También fueron incluidas en esta categoría aquellas descripciones citológicas donde la mayoría de las láminas mostraban una población de células foliculares normales, sin embargo, una minoría de grupos celulares mostraba una configuración papilar, agrandamiento nuclear, hendiduras nucleares, núcleos superpuestos y seudo inclusiones intranucleares. 
ISSN: 2215-7840, 6(1), enero-junio 2015, Osorio-Covo Carlos G., Grice-Reyes Juan M., Herrera-Banquez Karen, Caraballo-Gómez Cáceres

Pedro, Agresott-Marsiglia Rubén, Redondo-De Oro Katherine, Herrera-Sáenz Francisco

Bethesda $\mathrm{VI}$ - Maligno. Estudios citológicos que reporten hallazgos inequívocos de malignidad, consistentes con carcinoma papilar de tiroides, carcinoma medular de tiroides, carcinoma anaplásico de tiroides, carcinoma metastásico extratiroideo y linfoma de origen tiroideo.

Determinación del riesgo de malignidad. El riesgo de malignidad se obtuvo determinando el porcentaje de casos diagnosticados como malignos a partir de la biopsia en parafina para cada una de las diagnósticas del Sistema Bethesda para el reporte citopatológico de las lesiones de la glándula tiroides, de acuerdo con la siguiente formula: (casos malignos) / (casos malignos + casos benignos) x 100. (8). El hallazgo histopatológico incidental de un carcinoma papilar con un diámetro menor a 10 $\mathrm{mm}$, denominado microcarcinoma papilar, no fue considerado como maligno, a menos que el diagnóstico citológico previo fuera "sospecha de malignidad" o "maligno" (9).

\section{RESULTADOS}

La población de estudio fue definida en 161

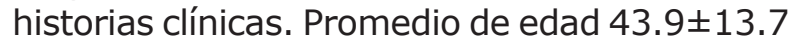
años, estuvo compuesta por 153 (95.0\%) mujeres y $8(4.9 \%)$ hombres. Únicamente 92 (57.1\%) diagnósticos citológicos fueron reportados utilizando el sistema Bethesda, mientras que $69(42.9 \%)$ no se ajustaban a dichos criterios. Los resultados obtenidos en la CACAF se muestran en la Tabla N01. El $11.8 \%$ de las CACAF fue obtenida mediante aspiración directa dentro de un quirófano, mientras que el $88.2 \%$ fueron obtenidas por un radiólogo utilizando guía ecográfica.

En 131 (81.3\%) pacientes, el resultado del estudio histopatológico fue de carácter benigno, mientras que en 30 (incluyendo los microcarcinomas papilares) el resultado fue de carácter maligno; dando un riesgo de malignidad atribuible total de $18.6 \%$ (30/161), y $14.9 \%$ (24/161) si se excluyen los microcarcinomas papilares (Tabla N02).

El riesgo de malignidad para cada una de las categorías de diagnóstico citológico se presenta en la Tabla N03 y la correlación de los diagnósticos citológicos e histopatológicos en la Tabla N04.

\begin{tabular}{|l|c|}
\hline \multicolumn{2}{|c|}{ TABLA No 1.} \\
DIAGNÓSTICOS CITOLógICOS \\
CATEGORIZADOS DE ACUERDO CON EL \\
SISTEMA BETHESDA
\end{tabular}

\begin{tabular}{|l|c|}
\hline \multicolumn{2}{|c|}{ TABLA No 2.} \\
\hline \multicolumn{2}{|c|}{ DIAGNÓSTICOS HISTOPATOLÓGICOS } \\
\hline Carácter benigno & $\mathrm{n}(\%)$ \\
\hline Bocio coloide multinodular & $131(81.4)$ \\
\hline Adenoma folicular de tiroides & $17(10.6)$ \\
\hline Tiroiditis linfocítica (Hashimoto) & $8(5.0)$ \\
\hline Carácter maligno & $30(18.6)$ \\
\hline $\begin{array}{l}\text { Carcinoma papilar de tiroides } \\
\text { (Variante clásica) }\end{array}$ & $13(8.1)$ \\
\hline $\begin{array}{l}\text { Microcarcinoma papilar de } \\
\text { tiroides (Variante clásica) }\end{array}$ & $6(3.7)$ \\
\hline $\begin{array}{l}\text { Carcinoma papilar de tiroides } \\
\text { (Variante folicular) }\end{array}$ & $4(2.5)$ \\
\hline $\begin{array}{l}\text { Carcinoma folicular de tiroides } \\
\text { (Variante clásica) }\end{array}$ & $3(1.9)$ \\
\hline Carcinoma medular de tiroides & $2(1.2)$ \\
\hline Carcinoma de células de hürthle & $1(0.6)$ \\
\hline Carcinoma anaplásico de tiroides & $1(0.6)$ \\
\hline
\end{tabular}

\begin{tabular}{|l|c|c|}
\hline \multicolumn{3}{|c|}{$\begin{array}{c}\text { TABLA NO 3. } \\
\text { RIESGO DE MALIGNIDAD EN LA } \\
\text { CITOLOGÍA OBTENIDA MEDIANTE } \\
\text { ASPIRACIÓN CON AGUJA FINA }\end{array}$} \\
\hline \multirow{2}{*}{ Diagnóstico citológico } & \multicolumn{2}{|c|}{ Casos malignos (\%) } \\
\cline { 2 - 3 } & $\begin{array}{c}\text { Incluyendo } \\
\mu \text { Ca }\end{array}$ & $\begin{array}{c}\text { Excluyendo } \\
\mu C a\end{array}$ \\
\hline $\begin{array}{l}\text { No diagnóstico o } \\
\text { insatisfactorio }\end{array}$ & $0(0.0)$ & $0(0.0)$ \\
\hline Benigno & $12(11.0)$ & $6(5.5)$ \\
\hline $\begin{array}{l}\text { Atipia folicular } \\
\text { de significado } \\
\text { indeterminado }\end{array}$ & $2(100.0)$ & $2(100.0)$ \\
\hline $\begin{array}{l}\text { Sospechoso de } \\
\text { neoplasia folicular }\end{array}$ & $3(14.2)$ & $3(14.2)$ \\
\hline $\begin{array}{l}\text { Sospechoso de } \\
\text { malignidad }\end{array}$ & $7(77.7)$ & $7(77.7)$ \\
\hline Maligno & $6(75.0)$ & $6(75.0)$ \\
\hline Total & $30(18.6)$ & $24(14.9)$ \\
\hline
\end{tabular}




\begin{tabular}{|c|c|c|c|}
\hline \multicolumn{4}{|c|}{$\begin{array}{c}\text { TABLA N }^{\circ} 4 . \\
\text { CORRELACIÓN ENTRE LOS DIAGNÓSTICOS CITOLÓGICOS E HISTOPATOLÓGICOS }\end{array}$} \\
\hline Diagnóstico citológico & N (\%) & Diagnósticos histológicos & N (\%) \\
\hline $\begin{array}{l}\text { No diagnóstico o } \\
\text { insatisfactorio }\end{array}$ & $12(7.4)$ & Bocio coloide multinodular & $12(100.0)$ \\
\hline \multirow{6}{*}{ Benigno: } & \multirow{6}{*}{109 (67.7) } & Bocio coloide multinodular & $86(78.9)$ \\
\hline & & Adenoma folicular de tiroides & $7(6.4)$ \\
\hline & & Microcarcinoma papilar de tiroides & $6(5.5)$ \\
\hline & & Tiroiditis de hashimoto & $4(3.6)$ \\
\hline & & Carcinoma papilar de tiroides (VC) & $4(3.6)$ \\
\hline & & Carcinoma papilar de tiroides (VF): & $2(1.8)$ \\
\hline \multirow{2}{*}{$\begin{array}{l}\text { Atipia folicular } \\
\text { de significado } \\
\text { indeterminado: }\end{array}$} & \multirow{2}{*}{$2(1.2)$} & Carcinoma papilar de tiroides (VC) & $1(50.0)$ \\
\hline & & Carcinoma medular de tiroides & $1(50.0)$ \\
\hline \multirow{6}{*}{$\begin{array}{l}\text { Sospechoso de neoplasia } \\
\text { folicular: }\end{array}$} & \multirow{6}{*}{$21(13.0)$} & Adenoma folicular de tiroides & $10(47.6)$ \\
\hline & & Bocio coloide multinodular & $7(33.3)$ \\
\hline & & Tiroiditis de Hashimoto & $1(4.7)$ \\
\hline & & Carcinoma papilar de tiroides (VC) & $1(4.7)$ \\
\hline & & Carcinoma papilar de tiroides (VF) & $1(4.7)$ \\
\hline & & Carcinoma de células de hürthle & $1(4.7)$ \\
\hline \multirow{6}{*}{$\begin{array}{l}\text { Sospechoso de } \\
\text { malignidad: }\end{array}$} & \multirow{6}{*}{$9(5.5)$} & Adenoma folicular de tiroides & $3(33.3)$ \\
\hline & & Carcinoma papilar de tiroides (VC) & $2(22.2)$ \\
\hline & & Carcinoma papilar de tiroides (VF) & $1(11.1)$ \\
\hline & & Carcinoma anaplásico de tiroides & $1(11.1)$ \\
\hline & & Bocio coloide multinodular & $1(11.1)$ \\
\hline & & Tiroiditis de Hashimoto & $1(11.1)$ \\
\hline \multirow{3}{*}{ Maligno: } & \multirow{3}{*}{$8(4.9)$} & Carcinoma papilar de tiroides (VC) & $5(62.5)$ \\
\hline & & Tiroiditis de hashimoto & $2(25.0)$ \\
\hline & & Carcinoma medular de tiroides & $1(12.5)$ \\
\hline
\end{tabular}

(VC): variante clásica.

(VF): variante folicular.

\section{DISCUSIÓN}

La revisión de los reportes citológicos permite observar que solo en la mitad de los casos, los hallazgos citológicos fueron categorizados de acuerdo al Sistema Bethesda. Ello indica la necesidad de difundirlo en el medio en el cual se realizó el estudio, ya que aporta mayor claridad para cirujanos y se refleja en la pertinencia de las indicaciones quirúrgicas (10). En el $42.9 \%$ los diagnósticos englobaban una terminología confusa, la cual como ha sido señalado puede entorpecer la comunicación y la toma de decisiones (11).

En las Tablas No 5 y No 6 se comparan los resultados del presente estudio con los ha- llazgos de la literatura. Se observó que la distribución de los casos según el Sistema Bethesda fue similar entre todos los estudios anteriormente mencionados. En el presente trabajo se encontró que 14 casos, a pesar de poseer diagnóstico citológico insatisfactorio, tuvieron seguimiento quirúrgico. Se puede atribuir la presencia en este grupo de diagnósticos insatisfactorios, a que en el centro hospitalario donde se realizó el estudio, algunas aspiraciones se realizaban sin guía ecográfica, como es la norma actual. Se observó que el $11.8 \%$ de las aspiraciones se realizaron sin guía, lo cual eleva las posibilidades de muestra citológica insatisfactoria. 


\begin{tabular}{|c|c|c|c|c|c|c|}
\hline \multicolumn{7}{|c|}{ TABLA No 5. } \\
\hline DISTRIBUCIÓN DE LoS DIAGNÓSTICOS CITOLóGICOS \\
\hline No diagnóstico & 7.7 & 18.6 & 7.0 & 10.4 & 5.0 & 1.2 \\
\hline Benigno & 67.7 & 59.0 & 66.0 & 64.6 & 64.0 & 87.5 \\
\hline AFSI & 1.2 & 3.4 & 4.0 & 3.2 & 18.0 & 1.0 \\
\hline SNF & 13.0 & 9.7 & 9.0 & 11.6 & 6.0 & 4.2 \\
\hline SM & 5.5 & 2.3 & 9.0 & 2.6 & 2.0 & 1.4 \\
\hline Maligno & 4.9 & 7.0 & 5.0 & 7.6 & 5.0 & 4.7 \\
\hline
\end{tabular}

AFSI: atipia folicular de significado indeterminado.

SNF: sospechoso de neoplasia folicular.

SM: sospechoso de malignidad.

\begin{tabular}{|c|c|c|c|c|c|c|}
\hline \multicolumn{7}{|c|}{$\begin{array}{l}\text { TABLA Nº } 6 . \\
\text { RIESGO DE MALIGNIDAD PARA CADA CATEGORÍA DE DIAGNÓSTICO CITOLÓGICO (*). }\end{array}$} \\
\hline Categoría de Diagnóstico & Presente estudio ${ }^{+}$ & Jo $(8)$ & Yassa (12) & Yang (13) & Nayar (14) & Mondal (15) \\
\hline No diagnóstico & 0.0 & 8.9 & 10.0 & 10.7 & 9.0 & 0.0 \\
\hline Benigno & 5.5 & 11.0 & 0.3 & 0.7 & 2.0 & 4.5 \\
\hline AFSI & 100.0 & 17.0 & 24.0 & 19.2 & 6.0 & 20.0 \\
\hline SNF & 14.2 & 25.4 & 28.0 & 32.2 & 14.0 & 30.6 \\
\hline SM & 77.7 & 70.0 & 60.0 & 64.8 & 53.0 & 75.0 \\
\hline Maligno & 75.0 & 98.1 & 97.0 & 98.4 & 97.0 & 97.8 \\
\hline
\end{tabular}

AFSI: atipia folicular de significado indeterminado.

SNF: sospechoso de neoplasia folicular.

SM: sospechoso de malignidad.

(*) Excluyendo microcarcinomas papilares

El riesgo de malignidad para las diferentes categorías diagnósticas del estudio se ajusta en parte con los riesgos mencionados en el Sistema Bethesda, pero difiere en algunos puntos de los riesgos reportados $(8,12-15)$. Entre tales puntos vale la pena resaltar que la categoría de diagnóstico citológico insatisfactorio tuvo un riesgo de malignidad de $0 \%$, similar a lo reportado por Mondal y cols, mientras que otros autores reportan un riesgo de malignidad de entre $9-11 \%$ (15).

El alto riesgo de malignidad encontrado en la categoría de diagnóstico citológico benigno fue considerable, un $11.0 \%$, al compararlo con el resto de la literatura, con rangos entre un 1 - 5\%, este comportamiento se pude atribuir a la alta incidencia de hallazgos malignos incidentales durante el análisis anatomopatológico de las piezas quirúrgicas, puesto que las lesiones catalogadas como microcarcinomas papilares (menores de 10 $\mathrm{mm}$ ), se escapaban de la evaluación ecográfica, y por lo tanto, no eran reportadas en la evaluación citológica de los aspirados.

Por último, los diagnósticos citológicos categorizados como malignos poseen un riesgo de malignidad de entre $97-100 \%$, pero en el presente estudio reporta riesgo de malignidad del $75 \%$. Los dos casos serían considerados como falsos positivos, estos correspondían a un diagnóstico histopatológico de 
tiroiditis de Hashimoto, en los cuales la tiroidectomía total es el procedimiento quirúrgico indicado.

La principal debilidad del estudio consiste en su linealidad retrospectiva ya que la calidad de las historias clínicas no permitió incluir un mayor número de variables. Tiene como fortaleza hacer notar el importante número de pacientes que acuden a la E.S.E. Hospital Universitario del Caribe con patologías del tiroides, lo que es insumo importante para realizar estudios de investigación con mayores alcances. El uso del Sistema Bethesda para el Reporte Citopatológico de las lesiones de la Glándula Tiroides (7), debiese ser adaptado por centros de diagnóstico, su estandarización puede me- jorar la forma cómo se diagnostican las lesiones de la glándula tiroides y por tanto conducir a un mejor enfoque en el manejo del paciente.

\section{CONCLUSIÓN}

El riesgo de malignidad deducido de los aspirados y categorizado de acuerdo al Sistema Bethesda para el Reporte Citopatologico de las lesiones de la Glándula Tiroides fue similar a los descrito en otros estudios.

CONFLICTOS DE INTERESES: ninguno que declarar.

FINANCIACIÓN: recursos propios de los autores.

\section{REFERENCIAS BIBLIOGRÁFICAS}

1. Mortensen JD, Woolner LB, Bennett WA. Gross and microscopic findings in clinically normal thyroid glands. J Clin Endocrinol Metab. 1955; 15(10):1270-80.

2. Ezzat S, Sarti DA, Cain DR, Braunstein GD. Thyroid incidentalomas. Prevalence by palpation and ultrasonography. Arch Intern Med. 1994;154(16):1838-40.

3. Román A, Restrepo L, Alzate C, Vélez A, Gutiérrez J. Nódulo tiroideo, enfoque y manejo. Revisión de la literatura. Iatreia. 2013; 26(2):197-206.

4. Wu HH, Jones JN, Osman J. Fine needle aspiration cytology of the thyroid: Ten years experience in a community teaching Hhospital. Diagn Cytopathol 2006;34(2):93-6.

5. Bongiovanni M, Cibas ES, Faquin WC. The role of thyroid fine needle aspiration cytology and the Bethesda system for reporting thyroid cytopathology. Diagn Histo. 2010;17(3):95-105.

6. Cooper DS, Doherty GM, Haugen BR, Kloos RT, Lee SL, Mandel SJ, y cols. Revised American Thyroid Association management guidelines for patients with thyroid nodules and differentiated thyroid cancer. Thyroid.2009;19(11):1167-214.

7. Cibas ES, Ali SZ. The Bethesda System for Reporting Thyroid Cytopathology. Am J Clin Patho. 2009;132(5):658-65.

8. Jo VY, Stelow ES, Dustin SM, Hanley KZ. Malignancy risk for fine needle aspiration of thyroid lesions according to the Bethesda system for reporting thyroid cytopathology. Am J Clin Pathol. 2010;134(3):450-6.

9. Cooper DS, Doherty GM, Haugen BR, Kloos RT, Lee SL, Mandel SJ, et al. Management guidelines for patients with thyroid nodules and differentiated thyroid cancer. Thyroid. 2006;16(2):109-42.

10. Richmond BK, O 'Brien BA, Mangano W, Thompson S, Kemper S. The impact of implementation of the bethesda system for reporting thyroid cytopathology on the surgical treatment of thyroid nodules. Am Surg. 2012;78(6):706-10.

11. Redman R, Yoder BJ, Massoll NA. Perceptions of diagnostic terminology and cithopathologic reporting of fine-needle aspiration biopsies of thyroid nodules: A survey of clinicians and pathologists. Thyroid. 2006;16(10):1003-8.

12. Yassa L, Cibas ES, Benson CB, Frates MC, Doubilet PM, Gawande AA, et al. Long-term assessment of a multidisciplinary approach to thyroid nodule diagnostic evaluation. Cancer. $2007 ; 111(6): 508-$ 16.

13. Yang J, Schnadig V, Logrono R, Wasserman PG. Fine-needle aspiration of thyroid nodules: A study of 4703 patients with histologic and clinical correlations. Cancer. 2007;111(5):306-15.

14. Nayar R, Ivanovic M. The indeterminate thyroid fine-needle aspiration: Experience from an academic center using terminology similar to that proposed in the 2007 national cancer institute thyroid fine needle aspiration state of the science conference. Cancer. 2009;117(3):195-202.

15. Mondal SK, Sinha S, Basak B, Roy DN, Sinha SK. The Bethesda system for reporting thyroid fine needle aspirates: A cytologic study with histologic follow-up. J Cytol. 2013;30(2):94-9. 